Proceedings

\title{
Electrospun Silk-Cellulose Composite Nanomaterials Using Ionic Liquid Regenerated Films ${ }^{\dagger}$
}

\author{
Ashley Rivera-Galletti ${ }^{1,2}$, Ye Xue ${ }^{1,3}$, Stacy Love ${ }^{4}$, David Salas de la Cruz ${ }^{4}$ and Xiao Hu ${ }^{1,2,3, *}$ \\ 1 Department of Physics and Astronomy, Rowan University, Glassboro, NJ 08028, USA; \\ riveragaa4@rowan.edu (A.R.-G.); xuey5@rowan.edu (Y.X.) \\ 2 Department of Chemistry and Biochemistry, Rowan University, Glassboro, NJ 08028, USA \\ 3 Department of Biomedical Engineering, Rowan University, Glassboro, NJ 08028, USA \\ 4 Department of Chemistry, Center for Integrative and Computational Biology, Rutgers University, \\ Camden, NJ 08102, USA; sw524@scarletmail.rutgers.edu (S.L.); ds1191@camden.rutgers.edu (D.S.d.1.C.) \\ * Correspondence: hu@rowan.edu \\ + Presented at the First International Conference on “Green" Polymer Materials 2020, 5-25 November 2020; \\ Available online: https://sciforum.net/conference/CGPM2020.
}

Citation: Rivera-Galletti, A.; Xue, Y.; Love, S.; de la Cruz, D.S.; Hu, X. Electrospun Silk-Cellulose Composite Nanomaterials Using Ionic Liquid Regenerated Films. Proceedings 2021, 69, 31. https://doi.org/ 10.3390/CGPM2020-07597

Published: 9 November 2020

Publisher's Note: MDPI stays neutral with regard to jurisdictional claims in published maps and institutional affiliations.

Copyright: $\odot 2020$ by the authors. Licensee MDPI, Basel, Switzerland. This article is an open access article distributed under the terms and conditions of the Creative Commons Attribution (CC BY) license (http://creativecommons.org/licenses/by/4.0/).

\begin{abstract}
Electrospinning is a widely used technique to draw recalcitrant biopolymer solutions into micro to nanoscale materials in a simple and economical way. The first focus of this research involved using ionic liquids as a non-volatile solvent for natural insoluble biopolymers such as silk and cellulose (or cellulose derivatives). Compared to traditional organic solvents, ionic liquids can dissolve biopolymers without altering the molecular weight of the biopolymer. In this study, 1ethyl-3-methylimidizolium acetate (EMIMAc) ionic liquid was used and the regenerated films were coagulated in baths of EtOH or water. The second focus of this research explored the dissolution of IL-regenerated composites into organic solvents and their electrospun composite nanomaterials. Various ratios of silk-cellulose bio-composite films regenerated from ionic liquids were used as the raw materials and sequentially dissolved/dispersed into a Formic Acid-CaCl 2 solution in order to initiate the electrospinning of silk-cellulose nanomaterials. Because of the variability of ionic liquids, the nanomaterials produced using this technique have unique and tunable properties such as large surface area to volume ratios and low structural defects. FTIR and SEM results suggest that the structure and morphology of the final nanosized samples becomes more globular when the biopolymer composition ratio has increased cellulose content. TGA results demonstrated that the electrospun materials have better thermal stability than the original films. This two-step electrospinning method, using ionic liquid as a non-volatile solvent to first dissolve and mix raw natural materials, may lead to extensive research into its biomedical and pharmaceutical applications in the future.
\end{abstract}

Keywords: ionic liquids; electrospinning; silk; cellulose; biomaterials

Figure 1 shows the FTIR spectra of EMIMAc generated silk-cellulose composite films and their regerated electrospun nanomaterials in the regions of $1500-1800 \mathrm{~cm}^{-1}$ and 800 $1200 \mathrm{~cm}^{-1}$. 



Figure 1. FTIR spectra comparing EMIMAc generated films and their electrospun nanomaterials in the regions of 1500$1800 \mathrm{~cm}^{-1}$ and $800-1200 \mathrm{~cm}^{-1}$. (1) and (2) depicts the FTIR spectra of EMIMAc generated films (MCC: Microcrystalline Cellulose; Cell $x$ : $x$ denotes the percentage of cellulose in the cellulose-silk composite. (3) and (4) depicts the electrospun nanomaterial samples as compared to MCC.

Institutional Review Board Statement: Not applicable.

Informed Consent Statement: Not applicable.

Data Availability Statement: The data presented in this study are available online at https://sciforum.net/paper/view/conference/7597 (accessed on 9 November 2020).

Acknowledgments: This study was supported by the National Science Foundation, DMR (1809541 and 1809354). The authors would like to thank Bailey Blessing from Rutgers-Camden University for her assistance. A special thanks to the Journal Polymers (MDPI). 